\title{
Evolvable Production Systems : MECHATRONIC PRODUCTION EQUIPMENT WITH PROCESS-BASED DISTRIBUTED CONTROL
}

\author{
Mauro Onori* José Barata**
}

\begin{abstract}
* Royal Institute of Technology, Dept. Of Production Engineering, Stockholm, Sweden (Tel: 46-8-7906637; e-mail: onori@ iip.kth.se).

** New University of Lisbon /UNINOVA, Quinta da Torre, Monte da Caparica, Caparica, Portugal(e-mail:jab@uninova.pt)
\end{abstract}

\begin{abstract}
Current major roadmapping efforts have all clearly underlined that true industrial sustainability will require far higher levels of systems' autonomy and adaptability. In accordance with these recommendations, the Evolvable Production Systems (EPS) has aimed at developing such technological solutions and support mechanisms. Since its inception in 2002 as a next generation of production systems, the concept is being further developed and tested to emerge as a production system paradigm. The essence of evolvability resides not only in the ability of system components to adapt to the changing conditions of operation, but also to assist in the evolution of these components in time such that processes may become self-evolvable, self-reconfigurable, self-tuning, self-diagnosing, etc. Characteristically, Evolvable systems have distributed control, and are composed of intelligent modules with embedded control. To assist the development and life cycle issues, a comprehensive methodological framework is being developed. A concerted effort is being exerted through European research projects in collaboration with European manufacturers, technology/equipment suppliers, and universities. After briefly stating the fundamental concepts of EPS, this paper presents current developments and applications.
\end{abstract}

Keywords: Evolvable Production Systems, Modularity, Distributed Control

\section{INTRODUCTION}

The major problems of manufacturing companies are all relate to uncertainty. First of all, it is very difficult for companies to predict the type and range of products that will have to be developed. The second uncertainty regards the production volumes and lifespan reached by these future products.

Evolvable systems, as a next generation of production systems, was first introduced in 2002 and has, since then, been developed and tested to emerge as a production system paradigm (see EUPASS, A3 projects). The essence of evolvability resides not only in the ability of system components to adapt to the changing conditions of operation, but also to assist in the evolution of these components in time such that processes may become self- $X, x$ representing a property of the system such as reconfigurable, tuning, diagnosing, etc.

According to the results attained by many roadmaps (ManuFuture [2005], ManVis [2007], FutMan [2005] and EUPASS [2008]), one of the most important objectives to be met by European industry is sustainability, which is multi-faceted: including economical, social and ecological aspects. The obvious conclusion to this holistic problem is that future manufacturing solutions will have to deal with very complex scenarios.
Evolvable Assembly Systems (Onori[2002], Barata et al [2006]) represents one of the paradigms proposed as an opportunity to solve such threats. NOTE: the relation to Holonic Manufacturing Systems (van Brussel [1998], Bussman et al [1999]) and Reconfigurable Manufacturing Systems will be described later.

Evolvable systems have characteristically distributed control, are composed of intelligent modules and are open in architecture. The technical and architectural aspects of the evolvable system development are supported by a comprehensive methodological framework. Evolvability being a system concept, it is envisaged to address every aspect of an assembly system throughout its life cycle, i.e., design and development, operation and evolution. The work has been and being implemented through large European research projects. Furthermore, integration of legacy subsystems and modules have been addressed in the methodology. It has, to date, resulted in several demonstrators and offered methodologies and architectures in support. This paper presents current developments and applications.

\subsection{BACKGROUND APPROACHES}

As defined in (ElMaraghy, [2006]) RMS incorporates principles of modularity, integrability, flexibility, scalability, convertibility, and diagnosability. These principles impose 
strong requirements to the control solution. In particular, centralized approaches become completely unsuited due to their intrinsic rigidity. Decentralised solutions must be considered that take into account the fundamental requirements of plugability of components, which includes the aspects related to dynamic addition / removal of components, as well as adaptation in the sense that the system does not need to be reprogrammed whenever a new module is added/ removed. This is a fundamental aspect behind any control solution approach to solve the defined requirements. Moreover, diagnosability also demands a decentralized approach, in particular if the manufacturing system is considered as a set of manufacturing components, each with diagnosis capability. The overall diagnosis of the system is obtained considering all the diagnosis information obtained from the individual modules. Due to these requirements, a particular and relevant aspect in the system being considered is the "intelligent" nature of its components, i.e., each component is considered as having computational power that will support individual diagnosability, dynamic plugability, adaptation, etc..

Therefore, the major challenge in the control solution is how to guarantee proper coordination and execution in a system in which both its components and working conditions can be dynamically changed. This is a challenge that needs a completely new approach and this is why in the context of EPS a solution based on concepts inspired from the Complexity Theory and Artificial Life is being developed. The next section covers what concepts from non traditional manufacturing research domains are being used to create truly dynamic control solutions.

Nevertheless, in the context of this paper it is important to clarify what are the big differences between the approach being proposed here and Holonic Manufacturing. The genesis of holonic manufacturing was very much a biological inspired approach and it was very close to the concepts of bionic and fractal. However, succeeding implementations along the years have drifted more and more from the original inspiration, and, in many aspects, the systems became more hierarchical.

Hence, the control approach to be developed in the context of EPS wants to go back to the basics, that is to say relying strongy on the original idea of considering each component as a distributed intelligent unit that may aggregate in order to create a complex system. In this context, concepts such as emergence and self-organisation become more and more important to be applied to new generation control solutions. Interestingly enough other researchers are proposing a selforganisation view in manufacturing enterprises (Tharumarajah,[2003]). However, true implementations of these new concepts within shop floor are still very few.

\subsection{EPS Concept}

Considering what was stated above, one may view Evolvable Production Systems (EPS) as a development of the Holonic Manufacturing Systems (HMS) approach; however, a closer looks reveals that, although there are similarities in the exploitation and implementation phases, the paradigms differ quite substantially in their perspective (or trigger issue), and that only EPS achieves fine granularity. By granularity it is considered the level of complexity of the component that compose a manufacturing system. For instance, when a line is composed of several cells and these cells are modules that can be plugged in and out, this is thick granularity. If, on the other hand, the components that can be plugged in or out are grippers, sensors, or pneumatic cylinders, this is fine granularity. This issue is in fact a very important one in terms of distinguishing the paradigms.

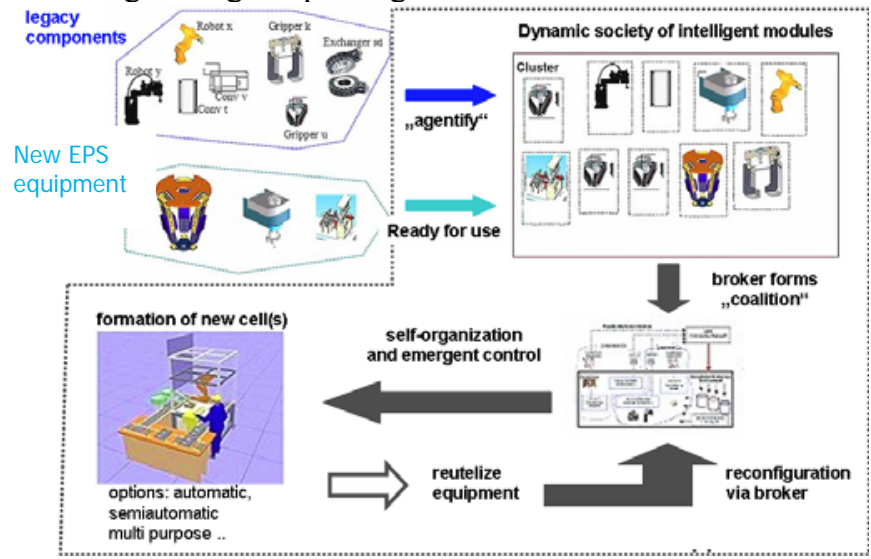

Fig.1.0 Basic Overview of EPS Approach

The main difference in the EPS paradigm is that it was created from a more dynamic, industrially-relevant perspective (trigger issue): EPS is mainly concerned with what occurs in a production system when a production change-over is called for; that is, whenever the current production system needs to undergo some change in its physical, control, or productivity layout. Such changes occur at ramp-up, product change-over, or demand surges. This is where the biological inspiration to EPS first makes itself apparent: it is change that drives the adaptability/evolution of the EPS systems, not the current or known scenarios. Furthermore, as will be detailed later, the adaptability is dictated by real evolvability principles such as "survival-ofthe-fittest" at algorithm level. This biological approach becomes even more evident when one studies the way modularity is achieved within EPS. In most approaches, modularity is set by either known mechanical subdivisions, or by taking the classical subdivisions that exist within manufacturing; for example, in reconfigurable assembly, the modules are most often set by the transport/handling/joining/placing/packaging processes. There is no biological link and the RMS and HMS paradigms tend to try to achieve a general, top-level solution. EPS is radically different in this respect as it will focus on the predicted and unpredictable changes that may occur within a very limited product range (genus). The first solution will be limited and specific, and may, if successful, gradually be applied to the associated product family (species). Hence EPS is not a generic solution but a specific approach that may be adopted by other "species" if its evolutionary capabilities denote a high rate of success.

Furthermore, EPS takes a hybrid and not top-down approach to the definition of its modules. The EPS modules are defined by precise sub-processes that have been identified for a given product range: the taxonomy of the sub-processes is very detailed and therefore results in fine granularity. This is a low-level approach, and gives modules with very optimised 
performance characteristics: process-oriented modules (Maraldo et al., [2006]). Note that since it is specific, and focuses on the given evolutionary demands of a product range and its exact sub-processes, it may also be closely linked to product design issues (discussed later). This is unique among current paradigms.

The third way in which one may associate EPS with biological systems is based on how its adaptability/evolvability is designed: based on many, process-specific elements/modules, the system control will be based on multi-agent systems that will autonomously capture emergent properties and act appropriately. The EPS systems will consist of finely granular solutions, with each module and/or unit possessing its own processing power. When these modules come together to form systems, cells or workstations, the aggregate skills of the units/modules will be greater than the sum of the individual skills: the emergence of new skills is precluded. Emergence, however, will also occur during the operational life-cycle of the modules, and will inevitably raise unwanted skills as well. Therefore, coping with emergent behaviour is a central issue within EPS.

\subsection{ENABLING RESEARCH DOMAINS AND CONCEPTS}

The main issue to be addressed in this section is describing the areas in which EPS control systems are getting inspiration to solve the requirements for adaptability at fine granularity. Numerous scientific domains investigating phenomena which EPS also exhibit have emerged in the last few years, which can provide helpful tools and valuable theoretical background to cope with the complexity of manufacturing systems. A more detailed definition may be found in an earlier publication (Barata et al, [2007].

\section{Complexity Theory}

Complexity Theory looks for simple causes leading to complex behaviors (Delic et al., [2006]). Complex systems are spatially and/or temporally extended non-linear systems with many strongly-coupled degrees of freedom. They are composed of numerous in themselves often simple elements and are characterized by collective properties. EPS consist of numerous equipment modules which are connected to each other and have multi-lateral interactions. Each of them has some degrees of freedom, which are constraint by other system parts. Together, the modules form a system with the desired global behavior.

Artificial Life

Taking natural life and its characteristics as an example, scientists attempt to create life-like behaviors with the capability of evolution on computers and other "artificial" media. EPS are very similar to artificial living systems. They have a modifiable structure, will exhibit some kind of selforganization, can adapt to their environment, and react to stimuli. They are capable of evolving according to the circumstances, namely in terms of equipment states, and can incorporate newly available technology. As any living organism, they will include efforts to keep themselves in a constant well-functioning state through self-surveillance and self-management - at least to a certain degree.

\section{Autonomic Computing}

Although at another level than the other areas described above, Autonomic Computing is a fundamental concept for EPS. The vision of Autonomic Computing (Kephart et al., [2003]) refers to the tendency of computers to become ubiquitous. Forming large networks and having complex and multiple interactions, they become increasingly difficult to manage. As a consequence, software will be designed to take care of itself. User interaction will be minimized and reprogramming avoided. Note that the more modules of fine granularity include computational power, the more is necessary to find new ways of coordination and automatic plugability, which is exactly what EPS want to address.

Agents

Depending on the context, an agent can be a human person, an association, an animal, or a piece of software, eventually connected to some hardware. The fundamental characteristics are identity, intelligence and the ability to act and react in order to persecute goals. Agents have at least a certain degree of autonomy and can compete or collaborate with others.

There are numerous successful experiences with agent-based systems in industry (Parunak, [2000], Monostori [2006]). Rockwell Automation even develops agent-based systems where the agents run inside the PLC itself (Mařík et al., [2005]) instead of on separate computers.

Self-Organization

Reasons for implementing self-organization in EPS are to minimize and facilitate user interaction, i.e. to hide complexity and increase system autonomy. Building and configuring a system composed of numerous entities with multi-lateral interactions is a highly complex task; the more autonomy the system has, the easier it gets for the user. Production systems tend to have many components of diverse nature which interact in many coupled ways. Agents need the capacity of organizing their collaboration themselves, in different forms and compositions, according to the needs, without passing through a central coordination point.

Emergence

Complex systems most often consist of at least two different levels: the macro-level, considering the system as a whole, and the micro-level, considering the system from the point of view of the local components. Local components behave according to local rules and based on preferably local knowledge; a representation of the entire system or knowledge about the global system functionality is neither provided by a central authority nor reachable for the components themselves. They communicate, interact with each other and exchange information with the environment. From the interaction in this local world emerge global phenomena, which are more than a straight-forward composition of the local components' behaviors and capabilities.

Typically, there is a two-way interdependence: not only is the global behavior dependent on the local parts, but their behavior is also influenced by the system as a whole. Emergent phenomena are scalable, robust, and fault-tolerant, i.e. insensitive to small perturbations and local errors as well as component failure, thanks to redundancy. They exhibit graceful degradation, meaning that there is no total breakdown because of minor local errors. 


\subsection{METHODOLOGY}

The EPS Methodology provides the references architecture, enablers, and modelling formalisms. In the following section brief description of the methodology is given. Note that a full description of the Reference Architecture is available through the EUPASS project framework (http://www.eupassfp6.org/pages/index.jsp).

\subsection{Modelling Formalisms}

The EPS formalised concepts (ontology) and definitions are represented using a set of descriptive tools such as :

- Definitions of the most important concepts: module, process, product, EPS module, skills, EPS system (which is a composition of modules), etc.

- Diagrams (UML, etc) where the interactions between the concepts defined are shown. This enables to show how the EPS architecture generates assembly systems. The interaction may show the global system behaviour

The domain ontology indeed captures the concepts in the system with their specifications (consensual semantic) i.e., what the concepts are and how they are related to each other in the domain. However, it does not capture the logic behind the relationships and the how's in the synthesis and functionality of the system.

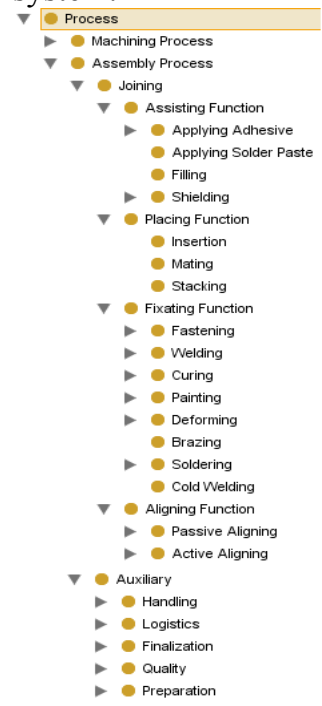

Fig.2.0 Partial view of the Assembly Process Taxonomy Taken from EPS Ontology.

\subsection{Reference Architecture, $R A$}

The EPS Reference Architecture (EPS_RA) describes the essential features of an Evolvable System which means the reference architecture specifies the necessary features that a system should have to be an evolvable system.

The reference architecture is composed of three main elements: Principles, Technical Positions and Templates.
Principles : EPS has two fundamental principles which lay the foundation and guide the development process of an evolvable system. These principles can be considered as a description of the core ideas of the evolvable system paradigm.

Principle 1: the most innovative product design can only be achieved if no assembly process constraints are posed. The ensuing, fully independent, process selection procedure may then result in an optimal assembly system methodology. Principle 2: Systems under dynamic conditions need to be evolvable, i.e., they need to have an inherent capability of evolution to address the new or changing set of requirements.

The enabling models include the development process model, the business model and the knowledge model. These models are constructed using the formalisms described above and most notably the EPS ontology.

The figure below depicts the first proposed EPS_RA using IDEF0. The is a simplified high level activity model showing the main activities in the development process and their input, output, control and mechanisms needed to generate or modify instances of the architecture,

The traditional top-down system design is feasible only in cases where the emergent behaviour is fully describable; (Ueda, [2001]). If emergent behaviour has to be investigated even at design and development stages, then a heterarchical or a network approach are the options.

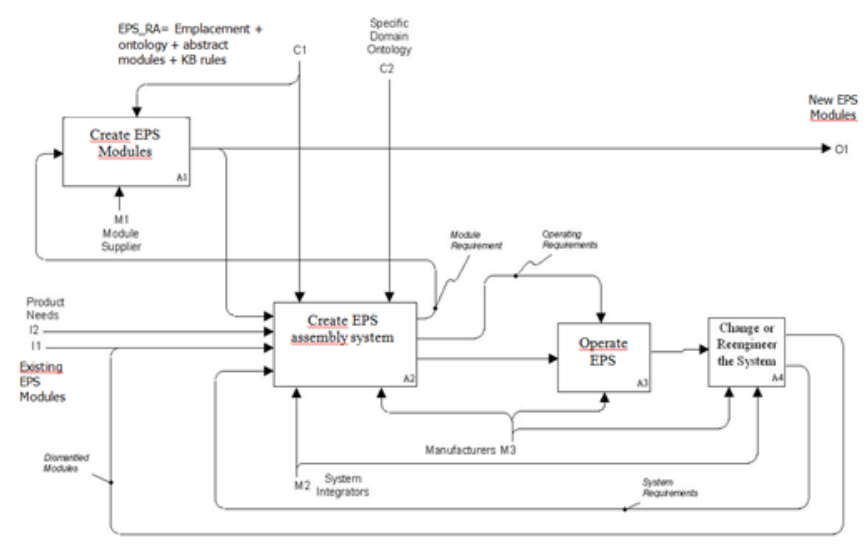

Fig.3.0 The simplified EPS_RA

Concepts that are formalised in the EPS Ontology are used to capture the stakeholders understanding of their own domain. The EPS knowledge model is thus a structured and formalised collection of such knowledge capturing representations of the domains. The main objective of the EPS knowledge model is to provide an environment that supports the development and operation of evolvable systems.

The domain knowledge captured using the EPS ontology and the EPS knowledge templates are the two entities used to develop the knowledge model. The knowledge models are used among other things how each module in a system should address for a new set of conditions.

The elements in the EPS knowledge model consists of the following knowledge domains: 
1. The enterprise knowledge domain - globalizes knowledge of the system and represents the business, organizational and global knowledge models captured. Enterprise knowledge enables environment recognition and maintenance of organizational associations.

2. The product knowledge domain - captures the knowledge related to product specification and design to assembly tasks.

3. The execution knowledge domain: capturing knowledge elements related to communications, planning and scheduling

4. The learning knowledge domain: containing knowledge elements that are used to incorporate case based reasoning.

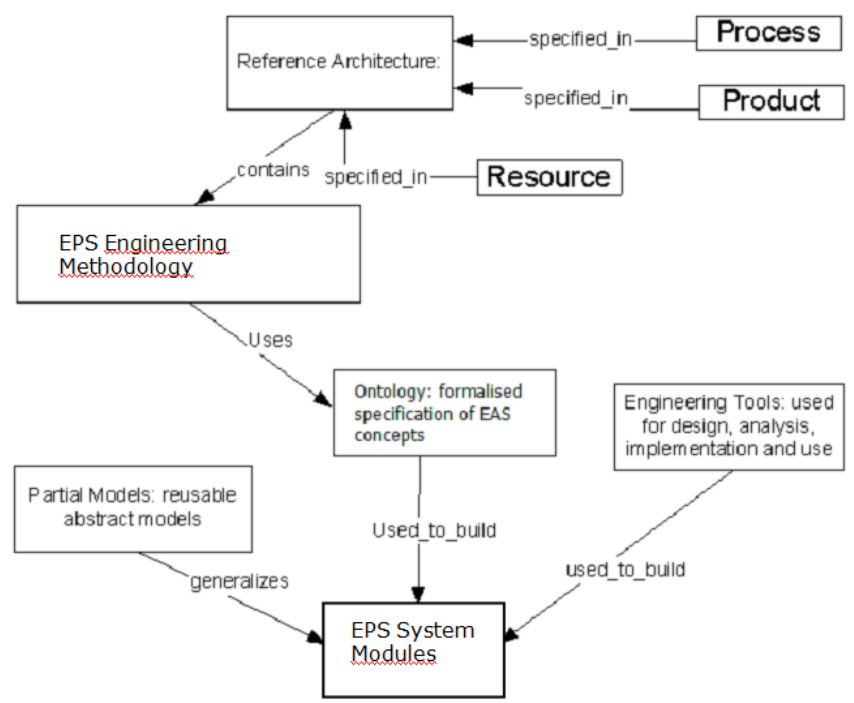

Fig.4.0 Basic aspects of the EPS Reference Architecture

The EPS RA is ultimately be viewed from different perspectives addressing the different concerns of the stakeholders. The stakeholders include:

- those who build the system (structure and communication views),

- those who use it (functional view),

- $\quad$ those who are concerned with control (control view).

The architecture should, therefore, address the concerns of every stakeholder, which gives rise to a multiple set of viewpoints concerning the requirements, expected outputs and controlling mechanisms. These include:

Functional View: address the concerns of the users of the system, and describe the functionality of the system, the process flows, quality, logistical issues.

Communication Views: address the concerns of how the data and information is to be represented, interpreted, recorded and transmitted.

Control views: address the concerns of the control system.

Structure Views: address the concerns of the system integrators, and maintenance.

These represent the background work that needs to be carried out in order to develop and establish an adequate EPS_RA. The actual implementation of this architecture, when considering the EPS modules and how they are to be integrated and formed into a system, will also be subject to a set of "views".

\section{PRACTICAL DEVELOPMENTS}

Initial evaluations were carried out in the test cell shown below (fig.5.0a). More industrially viable evaluations are currently being deployed within a new system being developed at KTH (fig.5.0b).

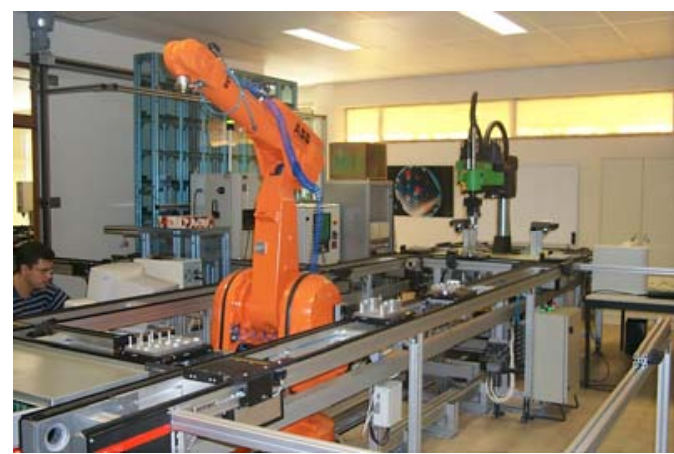

Figure 5.0a- The Evaluation Test Case Setup

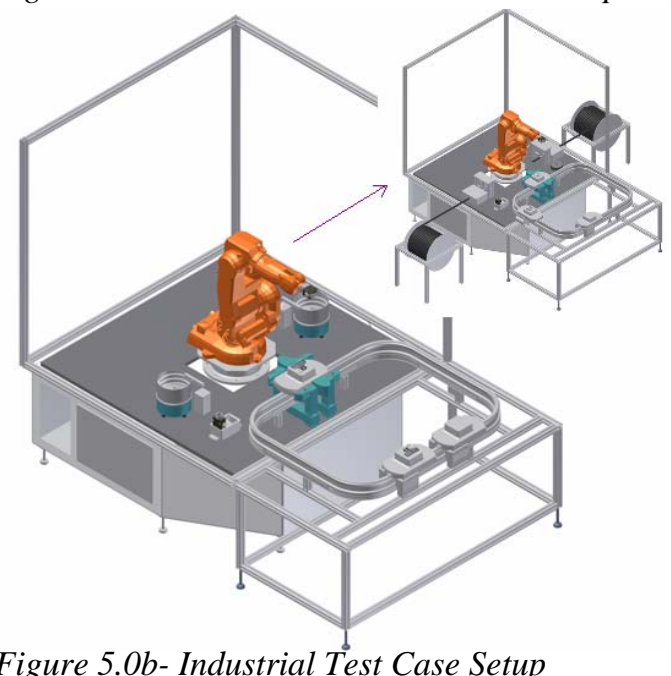

Figure 5.0b- Industrial Test Case Setup

At present the EPS paradigm is only just starting to take a practical form, and the control solution, ontologies, and methodologies only partially describe the most recent developments. These ideas are now being put into a real industrial scenario through the participation of Electrolux Home Products Italy SpA and UNINOVA. The layout given in figure $5.0 \mathrm{~b}$ is being setup for two industrial products (selfconfiguring \& reconfiguring).

The products are standard, off-the-shelf products. The project has now gone as far as to demonstrate that legacy equipment may be modified to Evolvable Production System (Adamietz, [2007]). An intelligent interface was developed out of an old Schunck gripper, as shown below, and the EUPASS project has now developed a full Evolvable Production System in Windisch, Switzerland. 


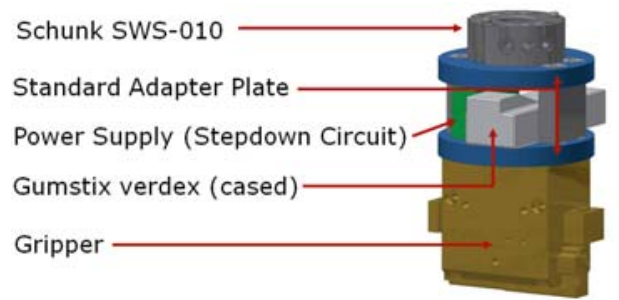

Fig.6.0 The Intermodular Receptacle

\section{CONCLUSIONS}

EPS, as with other similar approaches, offers great opportunities for attaining true agility and cost-effective, stepwise automation. The technologies for achieving this are available and there are several partners willing to partake in this endeavour; however, it is vital to point out that EPS does imply that the manner in which we develop and create projects for the development of assembly systems are radically changed, assuming a more synthesis-based approach.

In order to stimulate the further development and update of the paradigm and its applications, a collaborative webspace has been developed, in which the architecture, standards, equipment modules and other details are detailed and made accessible: www.Eas-Env.Org. This EAS Environment webspace is currently being expanded to include work from related projects.

The experience of this group is that there exists far too strong a resistance to such change in certain academic and industrial circles, and that the creation of new approaches and even new service sectors, all based on EPS and similar approaches, will have to prove their validity through even more elaborate industrial scenarios. Nevertheless, it must be said that at the end of the day it is not the most efficient or technologically advanced solution that may represent the future way of developing assembly systems, but the one that best paves the way to large-scale exploitation.

\subsection{REFERENCES}

- "Strategic Research Agenda-assuring the future of manufacturing in Europe"; Manufuture PlatformExecutive Summary, December 2005, EC

- "MANVIS, Manufacturing Visions-Integrating Diverse Perspectives into Pan-European Foresight"; FP6 Support Action, NMP2-CT-2003-507139

- "The Future of Manufacturing in Europe 2015-2020-The Challenge for Sustaninability"; FutMan, Institute for Prospective Technological Studies, European Commission Joint Research Centre, EUR 20705 EN

- "EUPASS Adaptive Assembly Roadmap 2015deliverable 1.5f"; Project Report-Public, Document 1.5f, EUPASS-Evolvable Ultra Precision Assembly, NMP-2CT-2004-507978; October 2008

- Onori, M. (2002a) In ISR2002 - 33rd International Symposium on RoboticsStockholm, pp. 617-621.

- Barata, J., Onori, M. and Frei, R. (2006) In ISIE'06 IEEE International Symposium on Industrial ElectronicsIEEE, Montreal - Canada.

- Van Brussel, H., Wyns, J., Valckenaers, P., Bongaerts, L. \& Peeters, P. (1998) Reference Architecture for
Holonic Manufacturing Systems: PROSA. Computers in Industry, 37, 255-274

- Bussmann, S. \& Mcfarlane, D. C. (1999) Rationales for Holonic Manufacturing; Second International Workshop on Intelligent Manufacturing Systems. Leuven, Belgium.

- Elmaraghy, H. A. (2006) Flexible and Reconfigurable Manufacturing Systems Paradigms. International Journal of Flexible Manufacturing Systems, 17, 261-276.

- Tharumarajah, A. (2003) A Self-organising View of Manufacturing Enterprises. Computers in Industry, 51, 185-196.

- Maraldo, T., Onori, M., Barata, J. \& Semere, D. (2006) Evolvable Assembly Systems: Clarifications and Developments to Date. CIRP/IWES 6th International Workshop on Emergent Synthesis. Kashiwa - Japan, 2006.

- Barata,J., Onori,M., Frei,R., Leitão, P. (2007); "Evolvable Production Systems - Enabling Research Domains"; Proceedings of the 2nd International Conference on Changeable, Agile, Reconfigurable and Virtual Production (CARV 2007), Toronto, Canada on 22-24 July 2007

- Delic, K. A. \& Dum, R. (2006) On the Emerging Future of Complexity Sciences. ACM Ubiquity, 7.

- Kephart, J. O. \& Chess, D. M. (2003) The Vision of Autonomic Computing. IEEE Computer, 0018-9162/03, 41-50.

- Parunak, H. V. D. (2000) Agents in Overalls: Experiences and Issues in the Development and Deployment of Industrial Agent-Based Systems. International Journal of Cooperative Information Systems.

- Monostori, L., Váncza, J. \& Kumara, S. R. T. (2006) Agent-Based Systems for Manufacturing. CIRP Annals, 55.

- Mařík, V., Vrba, P., Hall, K. H. \& Maturana, F. P. (2005) Rockwell automation agents for manufacturing. AAMAS. Utrecht, NL, ACM Press NY, USA.

- Ueda, K. (2001) Journal of Artificial Intelligence in Engineering, 15, 319-320.

- Adamietz, R.; "Development of an Intermodular Receptacle- A First Step in Creating EAS Modules"; MSC Thesis, KTH, Stockholm, Sweden, 2007. 